\title{
Optimizing and Modeling of Comprehensive Performance in Control Systems
}

\author{
Wenjin $\mathrm{Hu}^{1}$ Yingying $\mathrm{Su}^{2}$ Ling Nie $^{1}$ Yucheng Liu ${ }^{1}$ Taifu Li $^{1}$ \\ ${ }^{1}$ Dept. of Electronic Information Eng., Chongqing Univ. of Sci. \& Tech., Chongqing 400050, P. R. China \\ ${ }^{2}$ Dept. of Electronic Information and Automation, Chongqing Inst. of Tech., Chongqing 400050, P. R. China
}

\begin{abstract}
In control systems, it is usually short of quantified comprehensive performance index which results in the global optimization and parameter tuning problems. Aimed at these puzzles, in the paper, the comprehensive performance modeling and optimizing problems are studied based on the combined approach EK (Expert Knowledge) \&NN (Neural Network) and GA (Genetic Algorithm). Since skillful expert could exactly evaluate the control quality, EK was applied to quantify the comprehensive performance in PID control system. On the basis, the function of comprehensive performance related to adjustable parameters is modeled with $\mathrm{NN}$. When optimizing the NN function of comprehensive performance with GA, the globally optimal parameters were effectively searched. The experimental results reveal the validity of this modeling and optimizing approach. Therefore, with the combined approach EK \& NN and GA, the comprehensive performance in control systems can not only be availably modeled and also be globally optimized.
\end{abstract}

Keywords: Optimization, Comprehensive performance, Expert knowledge, Neural network, Genetic algorithm

\section{Introduction}

PID control is widely applied in process and motion control systems for its easy operation, fine robustness and high reliability [1]. In order to satisfy the industrial requirement of the stability, quickness, and accuracy, parameter tuning study in PID control has attracted more and more attentions from academic community and engineering field. In order to tune parameters, the explicit performance index is strongly demanded to evaluate the control quality. Generally speaking, there are two types of performance index [2]: (1) Single control index, such as overshoot, setting time, or subsidence ration, etc.; (2) Comprehensive control index, such as ISE, IAE, ITAE, etc. The former satisfies the single-objective optimization, and the latter realizes certain transient process optimization with minimizing integral error. To some extent, the two kinds of index can optimize control process, but when optimizing entire performance and obtaining the global optimal parameters, it is usually short of the comprehensive performance index. In this aspect, document [3] has proposed a novel method with multiobjective optimization when the indices are numerable. However, in actual control process the numbers of considerable indices are usually hundreds. If the feature space of comprehensive performance is only composed of some indices, it will result in the incomplete evaluation and not being globally optimized. Thus, the modeling between comprehensive performance and influencing parameters is one of the problems crying out for immediate solution.

In fact, a skillful expert can effectively evaluate the control quality at any case. Therefore, in the paper, the comprehensive performance will be quantified by EK. After that, the quantified control quality related to influencing parameters is capable to be modeled with NN approximating capability [4]. In this way, the explicit comprehensive quality function is obtained. On the basis of the object function, it is able to apply global searching algorithm GA [5] to optimize comprehensive performance and adjustable parameters. Consequently, the comprehensive performance in control systems is accurately modeled and optimized.

\section{Analog PID control system based on EWB software}

In analog PID control systems, the block diagram is generally shown in Fig.1.

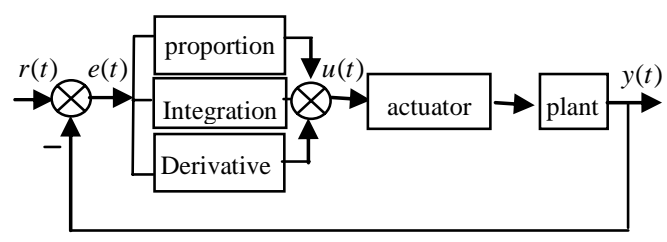

Fig. 1: The block diagram of general PID control systems.

The regulating rule of PID controller is: 


$$
u(t)=K_{p}\left[e(t)+\frac{1}{T_{i}} \int_{0}^{t} e(t)+T_{d} \frac{d e(t)}{d t}\right]
$$

Its transfer function is:

$$
G(s)=\frac{U(S)}{E(S)}=K_{p}\left(1+\frac{1}{T_{i} \cdot S}+T_{d} \cdot S\right)
$$

where $\mathrm{Kp} 、 \mathrm{Ti} 、 \mathrm{Td}$ are proportion, integration, and derivative coefficients, respectively. According to equation (1), (2), the control quality is directly determined by the combination $\mathrm{Kp}, \mathrm{Ti}$, $\mathrm{Td}$.

Here, global parameter tuning in PID control system is studied. Comprehensively considering the rational and effective verification, an analog PID control system is carried out in EWB (Electronics Work Bench) circuit analysis software in Fig.2. The block diagram and circuit diagram are respectively shown in Fig.3 and Fig.4.

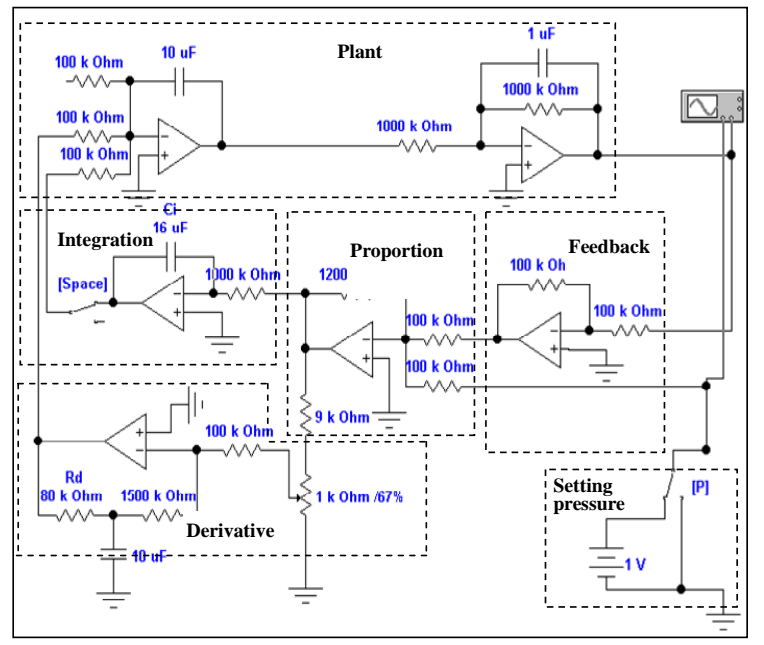

Fig. 2: PID control system in EWB software

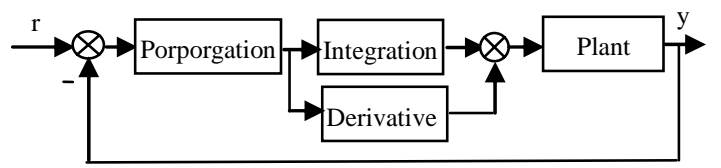

Fig. 3: The block diagram of PID control system in the paper

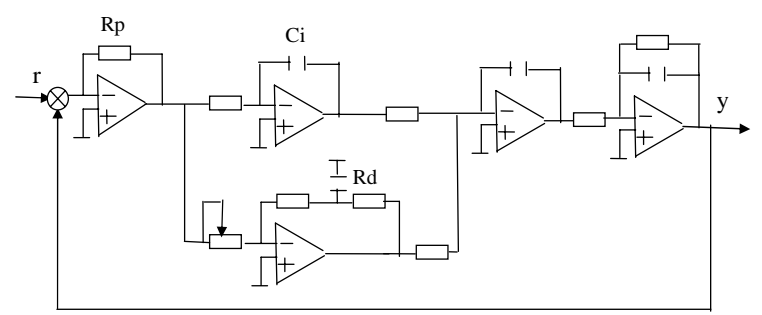

Fig. 4: The schematic diagram of PID control system

The transfer function in each part is given respectively:

Proportional component:

$$
\mathrm{G}_{\mathrm{p}}(\mathrm{S})=-2 \times 10^{-5} \cdot \mathrm{R}_{\mathrm{p}}
$$

Integral component:

$$
\mathrm{G}_{\mathrm{i}}(\mathrm{S})=-\frac{10^{-6}}{\mathrm{C}_{\mathrm{i}} \cdot \mathrm{S}}
$$

Derivative component:

$$
G_{d}(S)=-\left(1+10 \cdot R_{d} \cdot S\right)
$$

Controlled plant:

$$
G(S)=\frac{1}{S(S+1)}
$$

The entire transfer function of controller can be acquired as equation (7) and (8) with the combination of (3), (4), (5):

$$
\begin{aligned}
G_{C}(S) & =2 \times 10^{-5} \cdot R_{p} \cdot\left[\frac{10^{-6}}{C_{i} \cdot S}+1+10 \cdot R_{d} \cdot S\right] \\
u(t)= & 2 \times 10^{-5} \cdot R_{p} \cdot e(t)+\frac{2 \times 10^{-11} \cdot R_{p}}{C_{i}} \cdot \int_{0}^{t} e(t) d t \\
& +2 \times 10^{-4} \cdot R_{p} \cdot R_{d} \cdot \frac{d e(t)}{d t}
\end{aligned}
$$

In equation (8), the control quality for plant (6) is directly determined by the coefficients $K_{P}, K_{I}, K_{D}$. It equals that the control performance can be regulated by changing parameter combination of $R_{P}, C_{i}, R_{d}$ in Fig.2.

\section{Comprehensive performance modeling based on NN and EK}

There are various indices describing PID control quality, such as dynamic ones of overshoot $\sigma$, rise time $t_{r}$, setting time $t_{s}$, steady ones of steady error $e_{s s}$, and even many implicit indices. This leads to the difficulty in evaluating comprehensive performance in PID control system, which is in complex nonlinear relationship with many parameters. However, a skillful expert can easily sense and effectively evaluate it through observation and his experience. In this way, the comprehensive control quality can be quantified by EK. On the basis, $\mathrm{NN}$ is capable to approximate the complex mapping from influencing parameters to comprehensive evaluation to implement the comprehensive performance modeling.

Here, 3 examples of evaluating the control quality are given by expert in Fig.5. In this manner, the comprehensive performance is quantified with expert experience. 

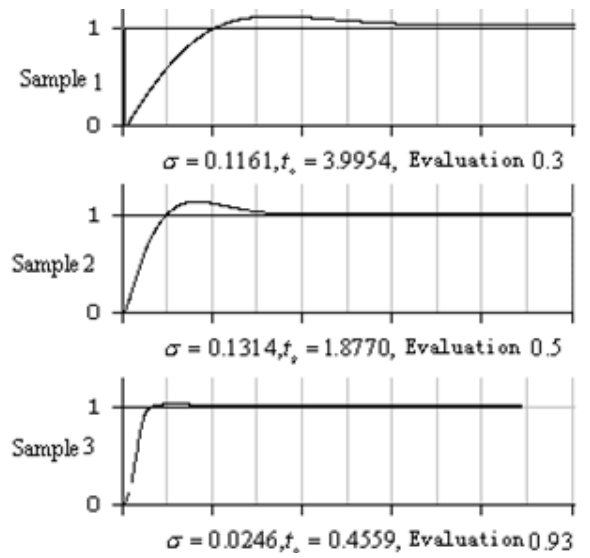

Fig. 5: Multi-objective decision with expert knowledge

The accuracy and generalization ability of NN directly lie on the completeness and effectivity of training data. So we adopt Uniform Design to acquire an initial training set of 10 experiment points [6]. Each parameter of $R_{P}, C_{i}, R_{d}$ is selected 10 levels forming a Uniform Design Table $U_{10}{ }^{*}\left(10^{8}\right)$ in Table 1 . The number in bracket is level of each factor.

Based on EWB software in Fig.2, experiments are done in turn. The expert correspondingly evaluates the control quality according to the response curve, and the results are given in Table 1. In Table 1, expert regards the bad response curve as zero score. Comparing the data in Table 1 , the optimal performance and parameters of PID control system are gained in the 6th experiment, where the response curve is shown in Fig.6. In order to enhance the accuracy of NN model, another 31 data pairs around the 6th experiment data are selected forming 41 training data pairs.

\begin{tabular}{|c|c|c|c|c|}
\hline No. & $\begin{array}{c}\mathrm{R}_{\mathrm{P}} \\
\left(10^{5} \Omega\right)\end{array}$ & $\begin{array}{c}\mathrm{R}_{\mathrm{D}} \\
\left(10^{4} \Omega\right)\end{array}$ & $\begin{array}{c}\mathrm{C}_{\mathrm{I}} \\
(\mathrm{uF})\end{array}$ & $E$ \\
\hline 1 & $2(1)$ & $5(5)$ & $12(7)$ & 0.27 \\
2 & $4(2)$ & $10(10)$ & $4(3)$ & 0.39 \\
3 & $6(3)$ & $4(4)$ & $18(10)$ & 0.50 \\
4 & $8(4)$ & $9(9)$ & $10(6)$ & 0.88 \\
5 & $10(5)$ & $3(3)$ & $2(2)$ & 0 \\
6 & $12(6)$ & $8(8)$ & $16(9)$ & 0.91 \\
7 & $14(7)$ & $2(2)$ & $8(5)$ & 0 \\
8 & $16(8)$ & $7(7)$ & $1(1)$ & 0 \\
9 & $18(9)$ & $1(1)$ & $14(8)$ & 0 \\
10 & $20(10)$ & $6(6)$ & $6(4)$ & 0 \\
\hline
\end{tabular}

Table 1: The experiment data of Uniform Design.

According to Kolmogorov theorem, a BP NN with one hidelayer could approximate any nonlinear continuous mapping $\mathrm{R}^{\mathrm{n}} \rightarrow \mathrm{R}^{\mathrm{m}}$ in $\mathrm{L}^{2}$ norm. Thus, we choose a multi-layer feedforward $\mathrm{NN}$ with one hide-layer, where inputs are adjustable parameter $R_{P}, C_{i}, R_{d}$ and outputs are expert evaluation $E$ of PID control quality. The transfer functions for hide-layer are tan-sigmoid. Since the expert evaluation is limited in interval $[0,1]$, the transfer functions for outputlayer are log-sigmoid. The nodes of neurons on hide-layer are

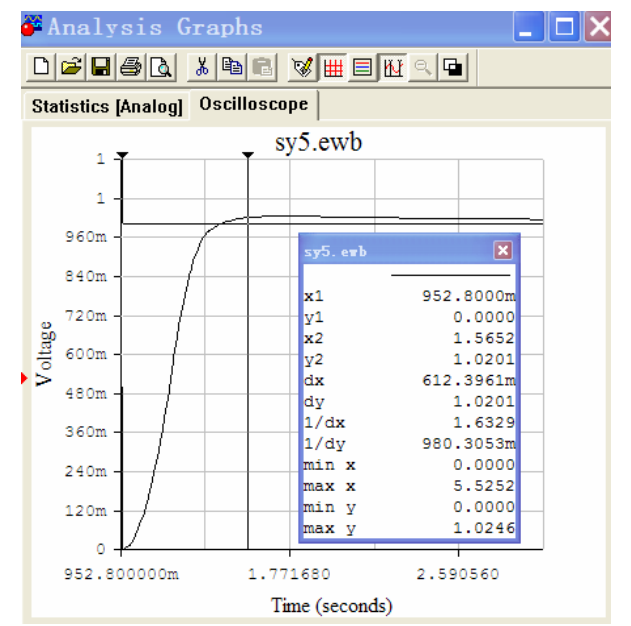

Fig. 6: The optimal response curve obtained with Uniform Design.

determined as 5 with preferable training effects. In this way, the structure of $\mathrm{NN}$ is determined as 3-5-1 in Fig.7.

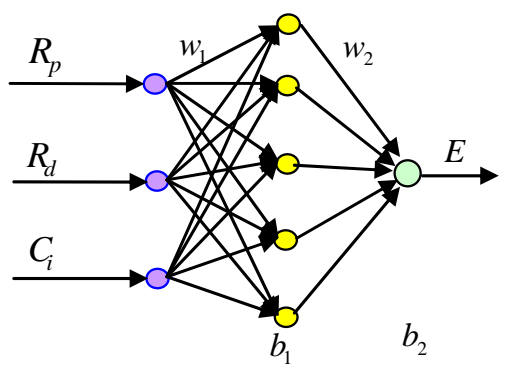

Fig. 7: The structure of NN mapping from $R_{p}, C_{i}, R_{d}$ to $E$.

In MATLAB NN Tools, training $\mathrm{NN}$ with "trainlm" algorithm, the approaching results and error curve are shown in Fig.8, the error square sum of trained NN is $\sum_{i=1}^{41} e^{2}=0.0041$. Besides, another 15 data pairs are used to test the generalization ability of trained NN, where the output and error curves are shown in Fig.9. Simulation results show that the accuracy and generalization ability of approaching model are both fine.
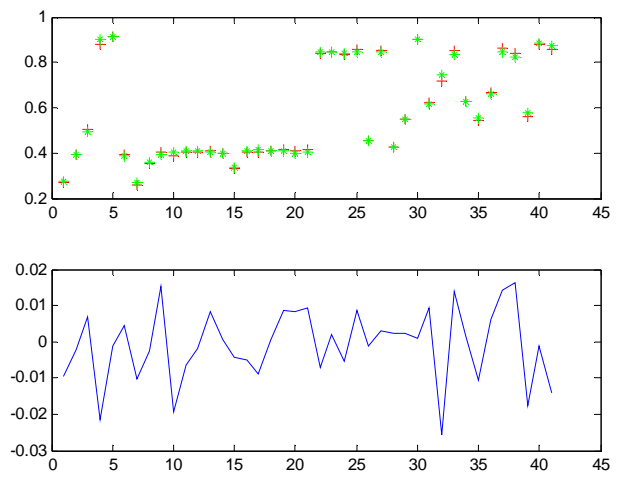

Fig. 8: Fitting results and error curve of NN. 

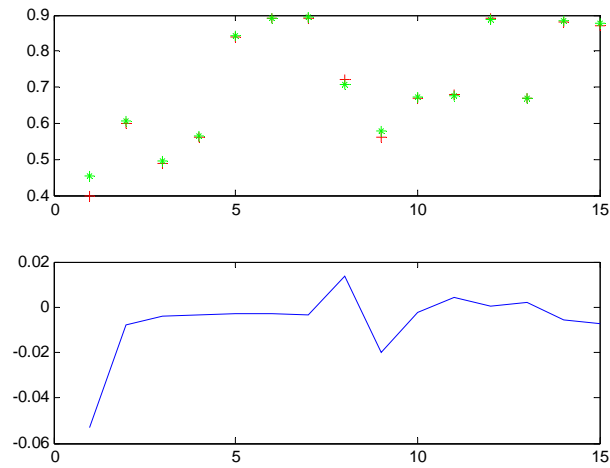

Fig. 9: The generalization ability of NN.

Thus, the model mapping from decision-making variables to comprehensive control quality is memorized in weights and thresholds. In this way, the comprehensive performance function is obtained as following:

$$
f=\frac{1}{1+e^{-x_{2}}}
$$

where $x_{2}=w_{2} \times y_{1}+b_{2}$

$$
\begin{aligned}
& y_{1}=\frac{2}{1+e^{-2 x_{1}}}-1 \\
& x_{1}=w_{1} \times\left[\begin{array}{l}
R_{p} \\
R_{d} \\
C_{i}
\end{array}\right]+b_{1}
\end{aligned}
$$

\section{Comprehensive performance optimizing for analog PID control system based on GA}

According to equation (9), the objective function of performance optimization with respect to adjustable parameters $R_{P}, C_{i}, R_{d}$ should be limited in a suitable interval which forms optimization problem (10):

$$
\min F=1-f\left(R_{p}, R_{d}, C_{i}\right)
$$

$$
\text { s.t. } \quad \mathbf{l b} \leq\left[\begin{array}{c}
R_{p} \\
R_{d} \\
C_{i}
\end{array}\right] \leq \mathbf{u b}
$$

GA can effectively solve many optimization problems referred to the Natural Selection and Random Search Algorithms. For problem (10), optimal value can be searched with MATLAB GA Toolbox. Furthermore, in order to avoid the discrete error of encoding/decoding, the descent learning algorithm in
MATLAB Optimization Toolbox will be further used to search the accurate optimal value and solutions.

The parameters in MATLAB GA Toolbox are settled as populations 40 , length of binary encoding 25 , crossover probability 0.7 and mutation probability $0.7 /(25 \times 3)=0.0093$. With the evolution of 50 generations, the optimal value $E$ and solutions $R_{p}, C_{i}$, $R_{d}$ of single objective function (10) are finally obtained as 0.9349 and $1.05 \times 10^{6} \Omega, 8.41 \times 10^{4} \Omega, 19.97 \mu \mathrm{F}$ in Fig.10.

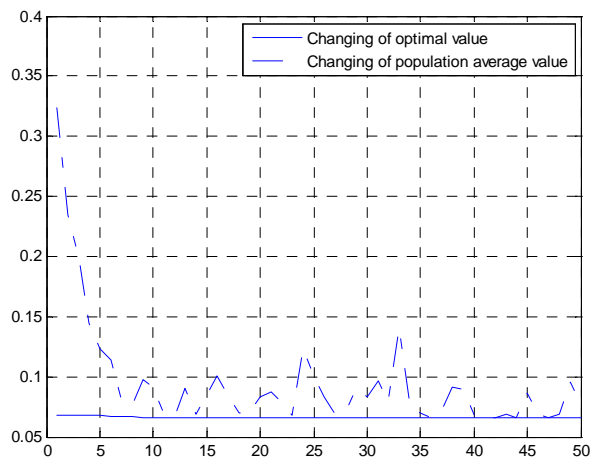

Fig. 10: The changing optimal value and average value with evolution.

After that, in order to enhance the accuracy, MATLAB Optimization Toolbox is applied to search perfect solutions when the above solutions $1.05 \times$ $10^{6} \Omega, 8.41 \times 10^{4} \Omega 、 19.97 \mu F$ were used as the initial point. In this way, the optimal value $E$ and solutions are exactly obtained as 0.9351 and $1.02 \times$ $10^{6} \Omega, 8.54 \times 10^{4} \Omega, 20 \mu \mathrm{F}$.

In the end, the final optimal solutions $R_{P}=1.02 \times 10^{6} \Omega, R_{d}=8.54 \times 10^{4} \Omega, C_{i}=20 \mu \mathrm{F}$ are resettled in PID control system. The control quality reveals really perfect in Fig.11, which is compared with that of Uniform Design in Fig.6. Moreover two performance indices of overshoot and setting time are compared in detail in Table 2, where the performance are all obviously improved compared with that in Uniform Design. Therefore, the application results further demonstrate that the modeling and optimizing method in the paper are valid.

\section{Conclusions}

In control systems, it is usually difficult to evaluate the vague comprehensive performance which results in the global optimization and parameter tuning problems. To these puzzles, selecting an analog PID control system as plant, the modeling and optimizing problems are effectively solved with the approach combined 


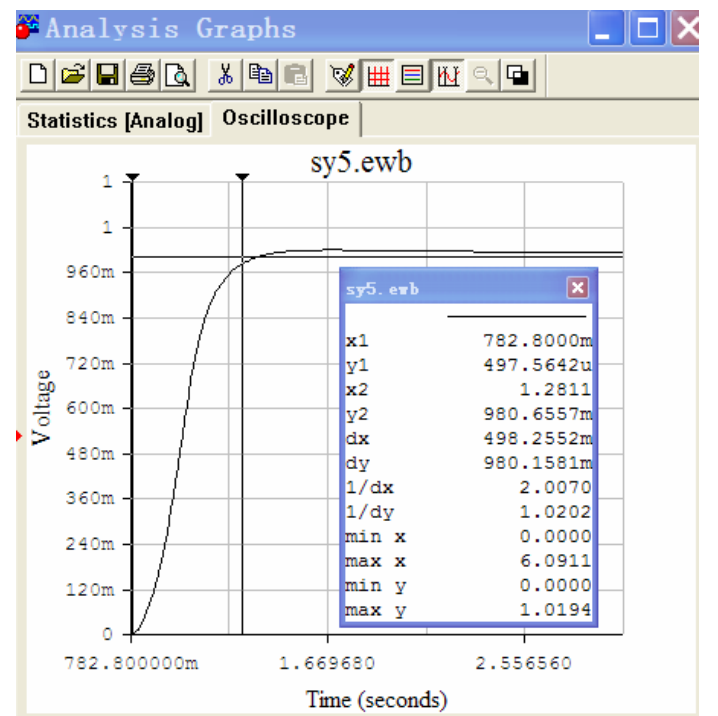

Fig. 11: The optimal response curve of PID control system obtained based on NN, EK and GA.

\begin{tabular}{|c|c|c|c|}
\hline \multicolumn{2}{|c|}{$\begin{array}{c}\text { Optimization } \\
\text { with Uniform Design }\end{array}$} & \multicolumn{2}{|c|}{$\begin{array}{c}\text { Optimization } \\
\text { with method in the paper }\end{array}$} \\
\hline $\begin{array}{l}\text { Decision-making } \\
\text { variables }\end{array}$ & $\begin{array}{r}\text { Perform } \\
\text { inde }\end{array}$ & $\begin{array}{l}\text { Decision-making } \\
\text { variables }\end{array}$ & \\
\hline $\begin{array}{ccc}R_{p} & C_{i} & R_{d} \\
\left.10^{6} \Omega\right) & (\mathrm{uF}) & (10 \Omega)\end{array}$ & $\begin{array}{c}\sigma \\
(\%)\end{array}$ & $\begin{array}{ccc}R_{p} & C_{i} & R_{d} \\
10^{6} \Omega & (\mathrm{uF}) & (10 \Omega)\end{array}$ & $\begin{array}{c}\sigma \\
(\%)\end{array}$ \\
\hline $\begin{array}{ll}1.2 & 16\end{array}$ & 2.46 & $\begin{array}{ll}1.02 & 20 \\
\end{array}$ & 1.94 \\
\hline
\end{tabular}

Table 2: The optimal results compared with the method in the paper and that in Uniform Design

EK\&NN and GA. With EK, the comprehensive performance is quantified. After that, NN with fine approximating ability is applied to model the quantified comprehensive performance with respect to influencing parameters. In the end, the optimal comprehensive performance and the solutions are effectively searched by GA. The experiment demonstrates the validity of the methodology. Therefore, the comprehensive performance in control systems can be accurately modeled with NN \& EK and optimized with GA.

\section{Acknowledgement}

The authors gratefully acknowledge the support of Chongqing Educational Committee Sci. \& Tech. Research Project (Grant No. KJ071411).

\section{References}

[1] J.K. Liu, Advanced PID control and MATLAB application, Publishing house of electronics industry, Beijing, 2003.

[2] J.S. Yu, Process control system and its application, China Machine Press, Beijing, 2003.

[3] X.H. Zhang, X.R. Zhu, J.G. Lin. Multi-objective optimization of PID regulators based on adaptive genetic algorithms, Systems Engineering and Electronics, 28: 744-746, 790, 2006.

[4] T.F. Li, Y.Y. Su and B.X. Zhong, Remodeling for fuzzy PID controller based on neural networks, Advance in Soft Computing, Springer-Verlag, 714-725, 2007.

[5] K.F. Man, K.S. Tang and S. Kwong, Genetic algorithms for control and signal processing, Springer-Verlag, London, 1997.

[6] T.F. Li, B. Guo and R.M. Deng, Intelligent control of some uncertainty complex system, Advances in Systems Science and Applications, 4: 231-237, 2004. 